\title{
Query-Adaptive Small Object Search using Object Proposal and Shape-Aware Descriptor
}

\author{
M.Venkatesh, Christeenajoseph
}

\begin{abstract}
To distinguish little and smooth organized items in a huge picture database. Dissimilar to well-known techniques investigating a rough picture equal pairwise comparability, the hunt is intended to abuse the likeness actions by the proposition equal. A successful diagram-based question extension system is intended to survey every one of these better-coordinated recommendations against every one of its neighbors inside a similar picture for exact confinement. Joined with a shapemindful element descriptor Edge Bob, a lot of increasingly shrewd edge-loads and hub utility measures, the proposed hunt technique can deal with differing view edges, enlightenment conditions, miss happening, and impediment proficiently. In picture recovery, include extraction of any information pictures in the dataset has been handled. The highlights of pictures have been grouped utilizing a sack of highlights. On the off chance that Order esteem is gotten least then the picture has been recovered. The proposed one can profit from the current in speed and precision and better execution.
\end{abstract}

Keywords:imageprocessing,segementation,matlab.

\section{INTRODUCTION}

Content-Based Image Retrieval is an application for recovering the pictures from a gigantic arrangement of picture databases dependent on the picture highlights, for example, shading, surface, what's more, some different traits. Here we take pictures to include as the folder to that picture and recover that specific picture. The greatest prompt application of the time content-based image retrieval in the script appears toward have been by, to represent his examinations into planned retrieval of images from a file by covering and form highlight. The term has since been generally used to depict the way toward recovering wanted pictures from an extensive gathering based on highlights, (for example, shading, surface, and shape) that can be consequently removed from the pictures themselves. The highlights utilized for recovery can be either crude or semantic, in any case, the extraction procedure must be overwhelmingly programmed. Recovery of pictures by physically allocated watchwords is unquestionably not CBIR as the term is commonly comprehended - regardless of whether the watchwords depict picture content. CBIR contrasts from established data recovery in that picture databases are basically unstructured, since digitized pictures comprise absolutely of varieties of pixel issues with any sort of picture preparing is the need to extricate valuable data from the crude information, (for example, perceiving the

Revised Manuscript Received on August 05, 2019.

M.Venkatesh, Department of ECE, Saveetha institute of medical and science, Chennai, Tamilnadu, India.

(E-mail: venkateshmaddineni1998@gmail.com)

Dr.Christeenajoseph, Associate professor, Department of ECE, Saveetha institute of medical and science, Saveetha University, Chennai, Tamilnadu, India.

(E-mail: christeena003@gmail.com) nearness of specific shapes or surfaces) before any sort of thinking about the picture's substance is conceivable. Picture databases in this manner vary in a general sense from content databases, where the crude material (words put away as ASCII character strings) has as of now been legitimately organized by the creator. There is no likeness level one recovery in a content database. CBIR draws a considerable lot of its plans as of the ground of image preparing then computer idea then stays observed by some as a subsection of that arena. It contrasts from these fields mainly through its accentuation on the recovery of pictures with wanted qualities since the accretion of noteworthy size. Picture making shelters a much additional wide field, including image upgrade, weight, show, and understanding. Though there are foggy parts, (for sample, thing acknowledgment through highlight examination), the refinement among normal image study and CBIR is generally reasonably clear. A precedent may brand this obvious. Numerous forces control currently use involuntary face greeting outlines. Such agendas might be used in one of two dissimilar ways. True off the bat, the image before the camera power be compared and a lonely person's folder greatest to check his or this woman character. For this condition, just two images are synchronized, a process barely any eyewitnesses would noise CBIR. Furthermore, the entire file strength is sought to discover the greatest resolutely directing images. This is a certifiable situation of CBIR. The way toward recovering wanted pictures from an expansive accumulation on the evidence of highlights, (for sample, shielding, shallow and form) that can be consequently separated from the pictures themselves. The highlights utilized for recovery can be either crude or semantic, however, the extraction procedure must be prevalently programmed. In regular Content-based picture retrieval frameworks, the visual material of the pictures cutting-edge the file is removed then portrayed through multi-dimensional element courses. The component courses of the descriptions popular the record structure a module database. To recover images, customers give the recovery framework by model images or outlined figures. The framework at that point vicissitudes these precedents into its inward portrayal of highlight courses. The similitudes/removes among the element courses of the review precedent or sketch and persons of the pictures in the file are formerly determined then recovery is performed with the guide of an ordering plan. The ordering plan gives a proficient approach to scan for the picture database. To alter the recovery procedure altogether to create perceptually and semantically increasingly significant recovery results.

Published By: 
The introduction picture is extraordinarily fit to programmed ordering for recovery. Frequently, introductions are conveyed with the guide of slides that express the author's topical organizing of the substance. Shots in which an individual slide shows up or is talked about relating to common units for fleeting picture division. Classically Twin Dispensation agenda integrates regarding images as twodimensional signs while put on efficiently set flag treatment plans to them. The interest for CBIR has developed as a result of the imprisonments inalienable popular metadatabased contexts, just as the extensive choice of conceivable habits designed for creative picture recovery. Printed data about pictures container be effectively sought utilizing existing origination, yet this expects persons toward actually portray each picture trendy the file. This container remain unreasonable used for vast files or else for pictures that observation cameras. He stands likewise imaginable near error pictures that use unique equal arguments popular their portrayals. Bases reliant on on ordering pictures in semantic courses similar "cat" by way of a subclass of "animal" flask dodge the miscategorization subject, so far determination need extra action through a user toward discover pictures that might remain "cats", yet areas it was vicarious an "animal". Numerous norms take remained shaped toward classify films, in any case, altogether still expression scaling and miscategorization issues.

\section{METHODOLOGY}

\section{Process with image using techniques:}

Picture planning is any kind of banner taking care of for which the data is an image, for instance, a photo before movie diagram. The crop of picture planning might remain also an double before a great deal of characters or limits connected to the double. Most picture dealing with techniques incorporate seeing the image as a twodimensional banner additionally, applying standard banner planning methodologies to it. Picture getting ready as a rule implies automated picture taking care of, anyway optical and basic picture planning in like manner are possible. Picture getting ready is immovably related to PC structures and $\mathrm{PC}$ vision. Picture planning is a procedure to change over an image into mechanized structure and play out a couple of undertakings on it, in order to get an improved picture or to isolate some supportive information from it. It is a kind of banner understanding where information is picture, as video packaging or photograph and yield may be picture or traits related with that image. Ordinarily Image Processing structure joins seeing pictures as two dimensional signs while applying viably set banner getting ready systems to them. Mechanized Image Processing Picture Processing Toolbox ${ }^{\mathrm{TM}}$ gives a broad arrangement of reference-standard figurings, limits, and applications for picture taking care of, examination, portrayal, likewise, estimation progression. You can perform picture examination, picture division, picture improvement, disturbance decline, geometric changes, and picture selection. Various device stash limits support multicore processors, GPUs, and C-code age. Picture Processing Toolbox supports a different game plan of picture types, continue shaped obviously, for example, persons from

including high powerful range, giga pixel objectives, embedded ICC profile, and tomographic. Discernment limits and applications let you explore pictures and accounts, take a gander at a district of pixels, adjust shading and distinction, make structures or histograms, and control regions of interest (ROIs). The toolbox reinforces work forms for getting ready, appearing, investigating extensive pictures. As a fundamental issue in the field of picture getting ready, picture recovery has been comprehensively inspected in the past two decades.

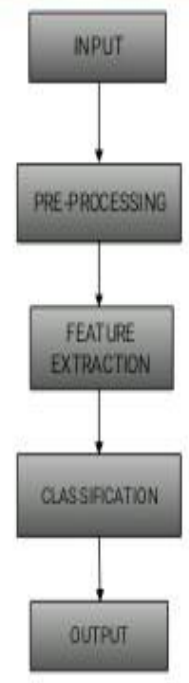

Fig 1:block diagram

It intends to remake the first astounding picture $\mathrm{x}$ from its corrupted watched adaptation $\mathrm{y}$, which is a commonplace not well presented straight reverse issue. Established regularization terms use nearby basic examples and are based on the suspicion that pictures are locally smooth aside from at the edges. Some delegate works in the writing are the all out variety (TV), half quadrature detailing, and Mumford-Shah (MS) models. These regularization terms exhibit high viability in protecting edges and recouping smooth districts. In any case, they generally smear out picture subtleties and can't manage fine structures, since they just adventure nearby insights, ignoring nonlocal measurements of pictures. Picture Preprocessing The probabilistic models endeavor to describe the key properties of an picture, in view of which imaging issue can be portrayed, defined and settled. For model, the objective of picture reclamation is to upgrade and to improve the appearance of a picture by assessing the first pixel esteems from the misshaped perception. An earlier demonstrate depicting measurements of both the commotion and the uncorrupted picture plays an fundamental job in this application. Because of high-dimensionality in spatial interactions, be that as it may, displaying the measurements of pictures is a testing task. The initial phase in lessening dimensionality is to make some disentangling presumptions in regards to the pixel bury connections. Another type of minimization utilitarian for settling picture backwards issue is detailed utilizing JSM 


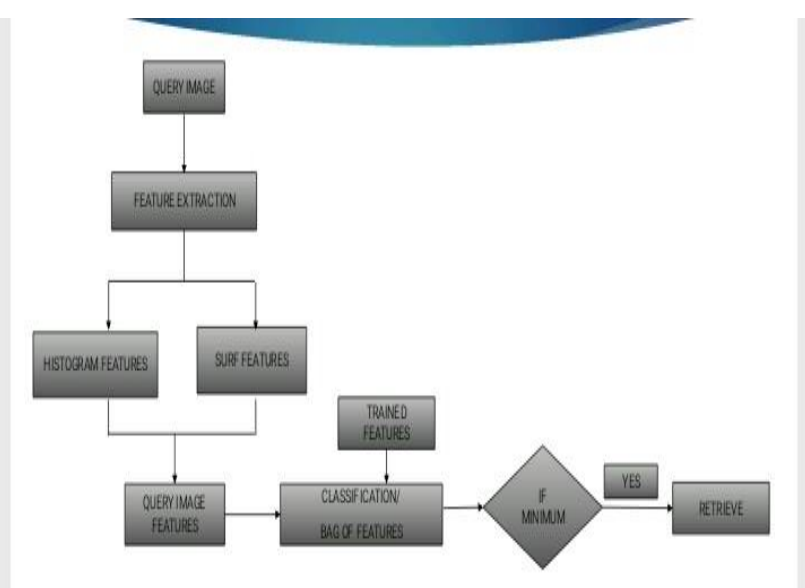

Fig 2:flow diagram

At last, in request to make JSM tractable and vigorous, another Split-Bregman based calculation is created to effectively comprehend the above seriously underdetermined backwards issue related with hypothetical evidence of assembly. Broatrials on picture inpainting, picture deblurring and blended Gaussian in addition to saltand-pepper commotion expulsion applications confirm the adequacy of the proposed calculation.

\section{EXISTING SYSTEM}

While there has been a lot of work on item hunt and picture recovery, the center has principally been on building up compelling models for the entire pictures, scenes, and items possessing a vast part of a picture. There has been a huge achievement seen in the space of picture recovery. A lot of ongoing works to focusses on tending to the issue of 3-D object recovery. In any case, coordinating and restriction for little articles like logos, protests commonly found in family units, and so forth.

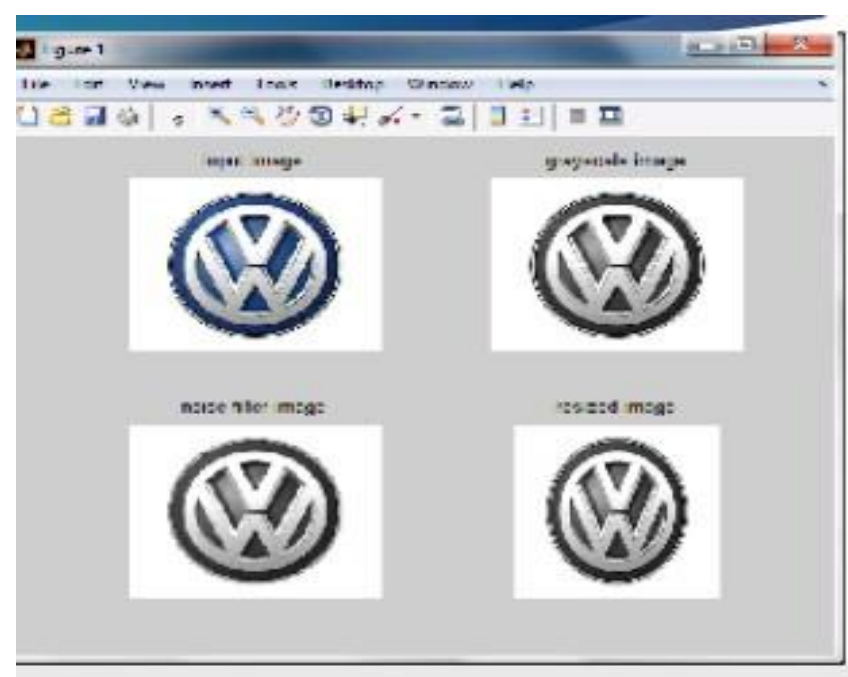

Fig 3:input image converted in gray scale image

In jumbled condition are as yet difficult. Some database pictures with instances of such objects of intrigue present in it. For instance, a particular class of visual items "logo" is graphically planned with hues, shapes,surfaces, maybe just as content additionally, following some particular spatial design. Logo pictures can likewise be obscured; the logo can possess just a little part in a picture with jumbled foundation and contrast essentially regarding relative twisting, commotion and impediment. The prominent pack of words (BoW) techniques additionally don't perform well in these situations and at last shape highlights end up being more discriminative and solid, which can agree to a great extent differentiating prerequisites of the issue details.

\section{PROPOSED SYSTEM}

The goal is to hold invariance in nearness of impediment, geometric and photometric changes, while in the meantime still stay delicate to neighborhood quirks to recognize the horrible altering and be very exact in its acknowledgment execution. Vigorous framework that can proficiently coordinate and precisely confine the examples of inquiry questions regularly possessing a little piece of a picture. The primary commitments are principally triple. form based component Edge BoF can catch adequate measure of worldwide shape data in nearness of changing enlightenment conditions, clamor, foundation mess, and so on., inside its structure. A proficient item level look system for coordinating and restriction Proposed Strategy a handle both the messiness concentrated genuine pictures and low quality double report pictures with equivalent productivi

\section{TEST AND RESULTS}

Arrangement difficult remains the period of execution, which went for safeguarding that the structure the whole thing correctly and gainfully before the live-action start. Challenging remains the path toward performing a package to learn a misstep. A better than the average trial remains unique that consumes a tall likelihood of discovery a screwup. A fruitful exam remains unique that responses a so far new botch. Challenging remains urgent for the accomplishment of the structure

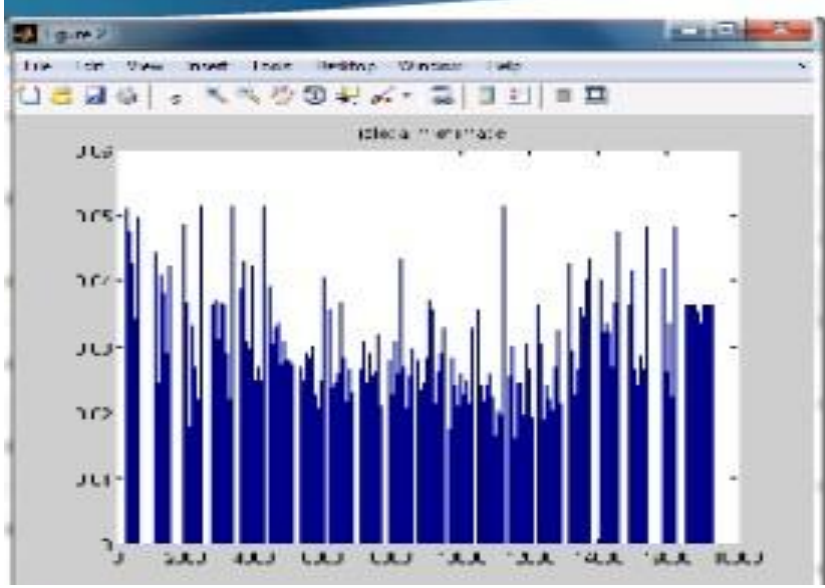

Fig 4:

Construction testing makes an authentic doubt that doubt altogether bits of the scheme remain precise, the target determination remain adequately practiced. The applicant construction remains at risk toward combination of tests-online reply, Capacity Road, retrieval and safety then usability examination. A movement of trials remain complete earlier 
the structure is set up aimed at the customer affirmation testing. Slightly fabricated thing can be attempted trendy unique of the going with ways. Significant the predefined work that a thing has remained planned to from, test can be coordinated to demonstrate each work is totally operational Unit testing is the attempting of each module and the joining of the general structure is done. Unit testing advances toward getting to be check attempts on the most diminutive unit of programming plan in the module. This is generally called 'module testing'. The modules of the structure are attempted autonomously. This challenging is finished in the midst of the software design himself. In this challenging stage, apiece perfect is seen toward work pleasantly as regard to the foreseen yield from the module. There are some endorsement checks for the fields. For point of reference, the endorsement check is practiced for affirming the information assumed through the customer anywhere together course of action and authenticity of the data enteredisconsolidated.

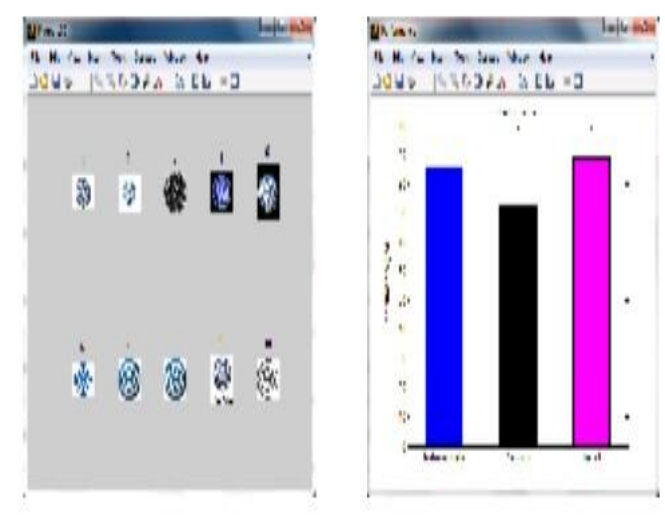

Fig 5: output

\section{OUTPUT TESTING:}

He remains particularly informal toward discovery botch then research the organization. In the wake of playing out the endorsement challenging, the ensuing phase remains crop getting some information near the association obligatory testing of the planned scheme later nope construction can remain valuable doubt he doesn't convey the obligatory yield trendy the exact game plan. The crop appeared or delivered through the scheme below idea. Here the yield configuration is careful in binary unique habits. Unique remains a shade and the other is a published location. The yield bunch happening the shade remains seen toward remain directly by way of the connotation was organized in the construction arrange as established through the client needs. Aimed at the published identical also harvest turns out by way of the decided necessities by the customer. Thus yield testing does not result in any relationship in the structure.

\section{CONCLUSION}

CBIR is utilized to look through an indicate picture from an expansive database. CBIR makes intuitive hunt of pictures from the database. At displayed this procedure is actualized by pack of highlights. They as appeared to be effective in improving exactness and speed on a basic trademark picture database. At long last the pertinent picture from databases has been recovered. As reported in the test results can give the best execution contrasted with different existing strategies. Therefore, the proposed plan can be considered as an exceptionally focused hopeful inshading picture recovery application.

\section{REFERENCES}

1 X. Wang, S. Qiu, K. Liu, and X. Tang, "Web image reranking using query specificsemantic signatures," IEEE Trans. Pattern Anal. Mach. Intell., vol. 36, no. 4, pp.810 823, Apr. 2014

2 Z. Zhong, J. Zhu, and S. Hoi, "Fast object retrieval using direct spatial matching,"IEEE Trans. Multimedia, vol. 17, no. 8, pp. 1391-1397, Aug. 2015.

3 Y. Jiang, J. Meng, J. Yuan, and J. Luo, "Randomized spatial context for object search," IEEE Trans. Image Process., vol. 24, no. 6, pp. 1748-1762, Jun. 2015.

4 P. Daras, A. Axenopoulos, and G. Litos, "Investigating the effects of multiple factors towards more accurate 3-d object retrieval," IEEE Trans. Multimedia, vol. 14,no. 2, pp. 374-388, Apr. 2012.

5 Y. Gao et al., "Less is more: Efficient 3-d object retrieval with query view selection," IEEE Trans. Multimedia, vol. 13, no. 5, pp. 1007-1018, Oct. 2011.

6 B. Gong, J. Liu, X. Wang, and X. Tang, "Learning semantic signatures for $3 \mathrm{~d}$ object retrieval," IEEE Trans. Multimedia, vol. 15, no. 2, pp. 369-377, Feb. 2013.

7 J. Meng, J. Yuan, J. Yang, G. Wang, and Y.-P. Tan, "Object instance search in videos via spatio-temporal trajectory discovery," IEEE Trans. Multimedia, vol. 18, no.1, pp. 116-127, Jan. 2016.

8 Y. Zhang, S. Zhang, W. Liang, and Q. Guo, "Individualized matching based on logo density for scalable logo recognition," in Proc. Int. Conf. Acoust. Speech Signal Process., 2014, pp. 4324-4328.

9 Y. Jiang, J. Meng, and J. Yuan, "Grid-based local feature bundling for efficient object search and localization," in Proc. IEEE Conf. Image Process., Sep. 2011, pp. 113116.

10 L. Yang, B. Geng, Y. Cai, A. Hanjalic, and X.-S. Hua, "Object retrieval using visual query context," IEEE Trans. Multimedia, vol. 13, no. 6, pp. 1295-1307, Dec.2011. 